\title{
On Intranode Impairments and Engineering Rules for an Optical Label Switching Router Supporting an FSK/IM Labeling Scheme
}

\author{
J. J. Vegas Olmos, I. Tafur Monroy, J. P. A. van Berkel, E. V. M. Verdurmen, J. G. L. Jennen, \\ and A. M. J. Koonen, Senior Member, IEEE
}

\begin{abstract}
This paper presents a study on intranode impairments and engineering rules for a label switching router supporting intensity modulated (IM) optical payload data signals labeled by using frequency-shift key (FSK) modulation. Engineering rules and design guidelines are presented regarding the choice of system parameters such as IM extinction ratio, FSK frequency deviation, alignment of optical filtering stages, label swapping, and node cascadability. The presented rules are derived from insights obtained from computer simulations and experimental validation for a $10-\mathrm{Gb} / \mathbf{s}$ IM payload signal.
\end{abstract}

Index Terms-Burst switching, optical communication, optical fiber communication, optical label switching (OLS).

\section{INTRODUCTION}

$\mathbf{T}$ HE EVER-increasing demand for bandwidth and flexibility in optical communication networks are driving the migration from circuit switching to packet switching leading in its turn to the study of intermediate scenarios. Those scenarios should be able to deliver high bandwidth under request and allow both circuit and packet switching network services. Optical packet switching (OPS) is a long-term strategy for network evolution that exploits fast switching techniques to provide greater bandwidth efficiency, flexibility, and functionality, and it offers finer granularity than circuit switching. However, OPS still require technological breakthroughs such as optical memories and buffering subsystems, and therefore, an intermediate technique for alleviating the migration to OPS is needed.

Optical label switching (OLS) is a promising technique for implementing IP packet routing and forwarding functions over wavelength division multiplexing (WDM) optical networks [1]-[3]. OLS reconciles the gap between the high data rate of data transmission over WDM links and the speed and complexity required to handle IP traffic at the core nodes of the network. OLS achieves this goal by labeling IP packets or burst of IP packets at moderate bit rates while the high-speed

Manuscript received April 3, 2006; revised June 12, 2006. This work was supported in part by The European Commission within the Information Society Technologies (IST) project Switching Technologies for Optically Labeled Signals (STOLAS).

J. J. Vegas Olmos, I. Tafur Monroy, J. P. A. van Berkel, E. V. M. Verdurmen, and A. M. J. Koonen are with the Communication Technology: Basic Research and Applications (COBRA) Institute of Technology, Technical University of Eindhoven, 5600 Eindhoven, The Netherlands (e-mail: j.j.vegas@tue.nl; i.tafur@tue.nl; jvberkel@dse.nl; e.v.m.verdurmen@tue.nl; a.m.j.koonen@ tue.nl).

J. G. L. Jennen is with the Bell Labs Research, Lucent Technologies, 1221 Hilversum, The Netherlands (e-mail: jeanjennen@lucent.com).

Digital Object Identifier 10.1109/JLT.2006.880604 payload data are kept in the optical domain from ingress-toegress node in the network. Several techniques for labeling the payload have been proposed so far [4]. Among them, a promising technique to label IP bursts (packets) is the so-called combined modulation format scheme, where the information of the label and the payload are conveyed simultaneously using a single optical carrier [5]-[10]. Within this specific approach, frequency-shift keying/intensity modulation (FSK/IM) is very promising due to its compact spectrum, simple label swapping, and scalability to high bit rates. This optical label switching (OLS) technique was studied in the recently completed project Switching Technologies for Optically Labeled Signals (STOLAS) [10]-[12].

This paper is organized as follows: In Section II, a generic OLS network architecture is described, followed by a description of the utilized labeling scheme. Furthermore, the core and edge node architecture studied in the STOLAS project are presented. In Section III, we describe the STOLAS lab trial experiments and focus on the intranode impairments of the OLS router. We identify the critical systems parameters and quantify via simulations and experiments their impact at node level performance. Section IV presents a set of engineering rules that can be used as design guidelines for an FSK/IM labeled switched IP over WDM system. In Section VI, the paper is concluded with conclusions.

\section{Network Architecture}

In this section, a reference OLS network architecture is introduced. The novel combined modulation format labeling scheme used in the STOLAS project is presented. The architecture of the edge and core nodes is explained.

\section{A. Generic Network Architecture}

The general architecture of an OLS network is depicted in Fig. 1.

In generalized multiprotocol label switching (GMPLS)based OLS network, bursts can be composed by assembling several IP packets (in ingress optical burst switching (OBS) nodes) and may contain several megabytes of data. For example, at the edge of a transport network, the packets from an access (or metropolitan) network are aggregated into bursts, buffered, and assigned a label in an ingress router. This label contains routing information, in a similar way to the electronic label-switched paths (LSPs) in the multiprotocol label 


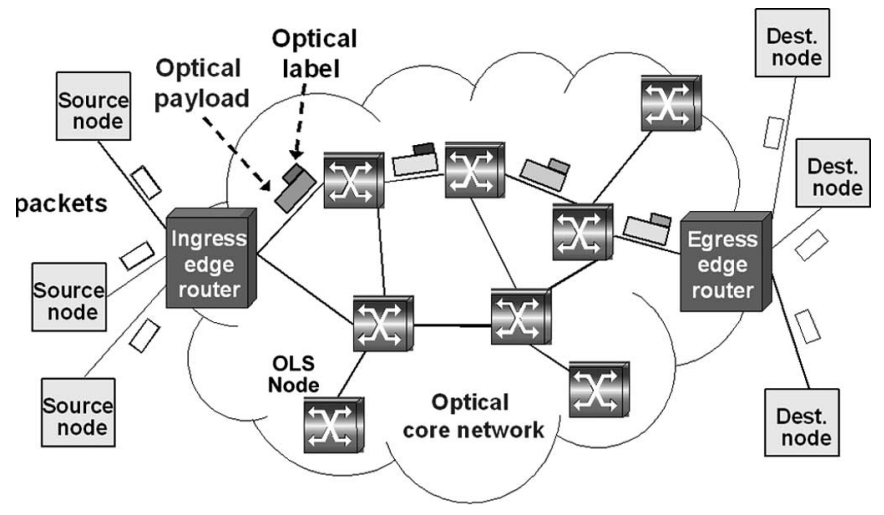

Fig. 1. General overview of a reference OLS network architecture.

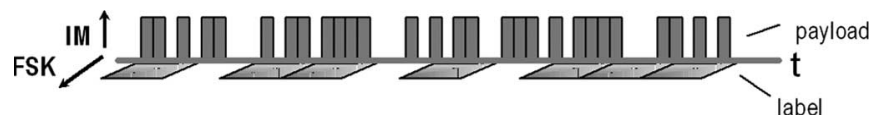

Fig. 2. Optical FSK/IM labeling scheme.

switching (MPLS) protocol. The payload is then transmitted using a certain wavelength channel. At the core node, the label is read and processed, and a new one is created accordingly and attached to the payload. The packet will be conveyed in that way through the entire network until it arrives to the final egress node. There the labeled payload will be directed to its final destination node. During this process, only the optical label will be processed while the high-speed payload data are kept in the optical domain, which results in increased throughput of the routing nodes.

\section{B. Labeling Scheme}

The intensity and frequency of an optical carrier can be visualized as defining a two-dimensional space. When the payload is carried by intensity variations, one may code the label information in the orthogonal dimension in this space, using FSK, as shown schematically in Fig. 2. In principle, this method permits label coding without a significant increase of the optical bandwidth of the signal [13].

The optical field of a labeled packet $x(t)$ can be described as

$X(t)=E_{0} \sqrt{1+m \cdot P(t)} \exp \left(i\left(\omega_{0} t+\frac{\Delta \omega_{\mathrm{f}}}{2} \int_{-\infty}^{t} L(t) d t\right)\right)$.

Here, $E_{0}$ represents the field amplitude, $P(t)$ the payload data, $L(t)$ the label data, $\omega_{0}$ the optical frequency, $\Delta \omega_{\mathrm{f}}$ the frequency deviation value, and $m$ the IM depth. Both $P(t)$ and $L(t)$ are assumed to have square elementary data waveforms, varying in the range of $[-1,1]$. The modulation index $m$ is related to the extinction ratio (ER) of the IM signal, as expressed in the next equation:

$$
\mathrm{ER}=\frac{1}{1+m}
$$

An optical FSK labeling technique is scalable to high bit rates and allows upgrades in the payload bit rate without severe changes in the labeling part of the system. Moreover, it allows

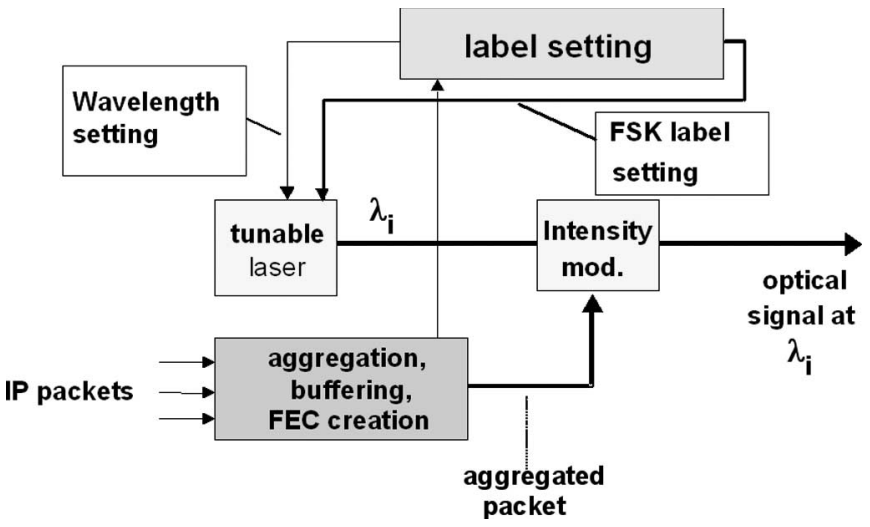

Fig. 3. Edge node architecture. FEC: forward equivalence class.

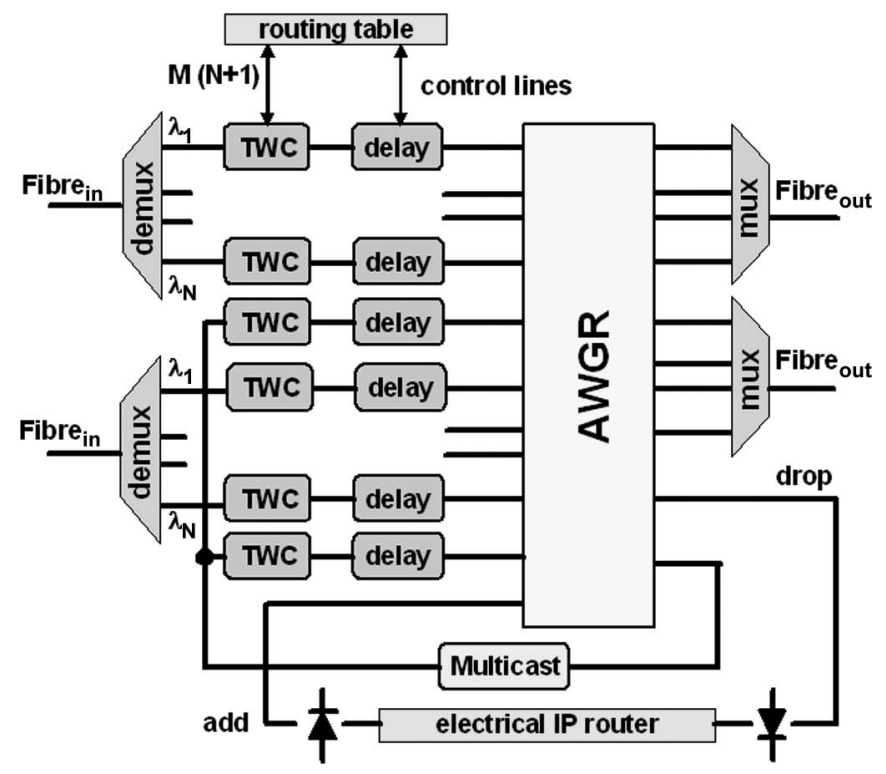

Fig. 4. Core router architecture with an arrayed waveguide grating router (AWGR) as routing element. TWC: tunable wavelength converter.

for label information to be easily extracted at a lower bit rate employing for low-cost electronics in the network nodes. Furthermore, the label and data payload are uncoupled regarding timing, and thus, only synchronization at packet level is needed (not at the bit level).

The disadvantages of a combined modulation scheme are mainly related to the use of compromised value of the ER of IM payload data. A limited ER on the payload is needed in order to minimize crosstalk between payload and label [14]. Moreover, phase (or frequency) to intensity conversion, due to dispersion, optical filtering effects, or chirp effects in the fiber links during propagation and inside the optical routing nodes can lead to a degradation of both the label and the payload performance. Section IV presents engineering rules for implementing scalable FSK/IM label swapping systems.

\section{Edge and Core Nodes}

As described in previous sections, the high-speed payload data are transmitted using IM format, while the label data are conveyed on the same optical carrier by FSK modulation. At the edge router (see Fig. 3), the aggregation, buffering, and possibly 


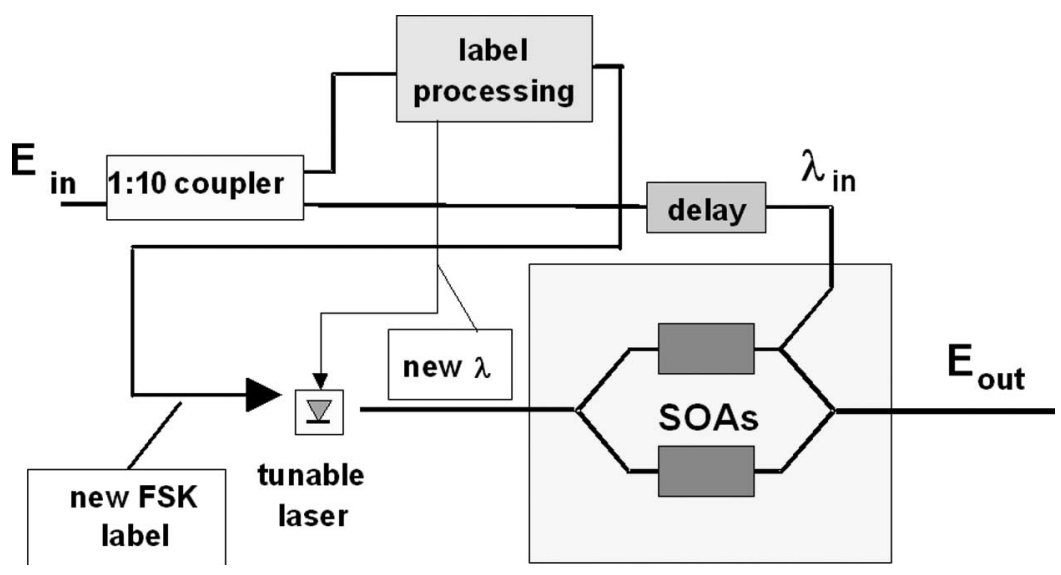

Fig. 5. Generic optical TWC.

the application error correction codification of incoming IP packets sharing the same destination or quality of service (QoS) are performed.

The packet routing information is sent to an electronic stage, which will generate the FSK label by frequency modulation of a tunable laser source (TLS). The TLS will then generate a signal at certain wavelength channel containing the FSK label information. The payload information is further IM, superimposed to the FSK, obtaining a combined FSK/IM label packet at the output of the edge router.

The generic architecture of core routing node is depicted in Fig. 4.

In this core node, the incoming channels are demultiplexed, and send into a tunable wavelength converter (TWC), which will replace the old label with a new one. Within the STOLAS project, an arrayed waveguide grating (AWG)-based labelcontrolled switch that efficiently integrates widely tunable lasers and semiconductor optical amplifier-Mach-Zehnder interferometer (SOA-MZI) wavelength converters was developed. Furthermore, multicasting of packets is required for implementing services such as video distribution and video conferencing and for realizing virtual private networks [15]. The multicasting function at the core router is obtained by feeding packets from a dedicated multicast output port of the AWG back to the input ports that are appropriately selected by controlling the TWC, followed by routing through the AWG.

A key building block for the implementation of the aforementioned optical labeling technique is the label swapper module that is in charge of extracting and reinserting the optical labels from each incoming burst. This label swapper is depicted in Fig. 5.

As shown in Fig. 5, to perform label swapping, a fraction of the incoming signal is tapped for optoelectronic label processing. The remaining part of the signal is input into an SOAMZI for wavelength conversion by intensity-driven cross-phase modulation. In the same SOA/MZI, label removal and new label insertion takes place. Replacing the label is easy because the FSK label information is lost during the wavelength conversion in the SOA-MZI. Therefore, only the payload information will be copied onto the output wavelength of the SOA-MZI, which is FSK modulated with the new label information. Modulating the phase section of a single grating-assisted coupler sampled

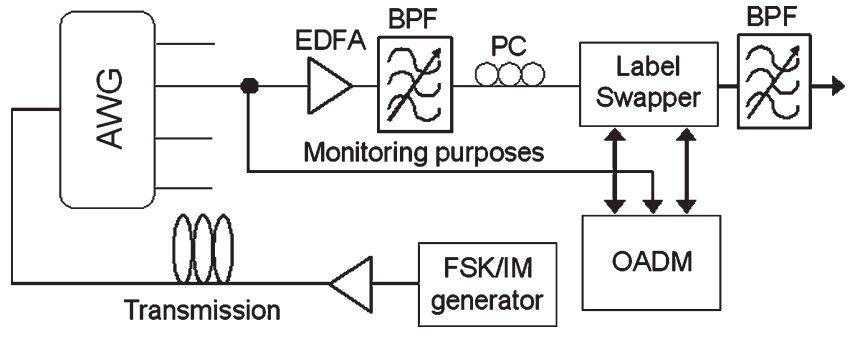

Fig. 6. STOLAS lab-trial setup. EDFA: erbium-doped fiber amplifier. AWG: arrayed-waveguide grating. PC: polarization controller. TLS: tunable laser source. IM: intensity modulator.

TABLE I

STOLAS GRID

\begin{tabular}{ccc}
\hline \hline Wavelength & Wavelength [nm] & Frequency [GHz] \\
\hline 0 & 1555.75 & 192.7 \\
1 & 1557.36 & 192.5 \\
2 & 1558.98 & 192.3 \\
3 & 1560.61 & 192.1 \\
\hline \hline
\end{tabular}

reflector (GCSR) [16]-[18] tunable source enables FSK generation, as we report below, and this signal is used as the new output wavelength for the SOA-MZI converter. In this way, the FSK label is swapped, and the payload data are ready for the next hop transmission. When followed by a passive AWG wavelength router, the new wavelength will determine the switching path inside the node.

\section{STOLAS LAB TRIAL}

The experimental setup of the STOLAS project demonstrator is depicted in Fig. 6.

A one-by-four AWG was set as a routing element. The four wavelength channels used during the experiments are shown in Table I. The labeled signals were generated using TLSs with FSK and IM generation capabilities.

The payload signal was generated using a $10-\mathrm{Gb} / \mathrm{s}$ pseudorandom bit sequence (PRBS) $2^{31}-1$ data stream, while the label was a $50-\mathrm{Mb} / \mathrm{s}$ signal containing a 16-bit label format, including the hexadecimal word "10." The label format is depicted in Fig. 7. A 4-bit unique word is used for localization 


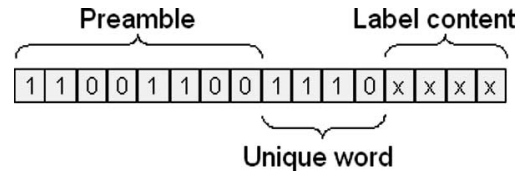

Fig. 7. A 16-bit STOLAS label composition.
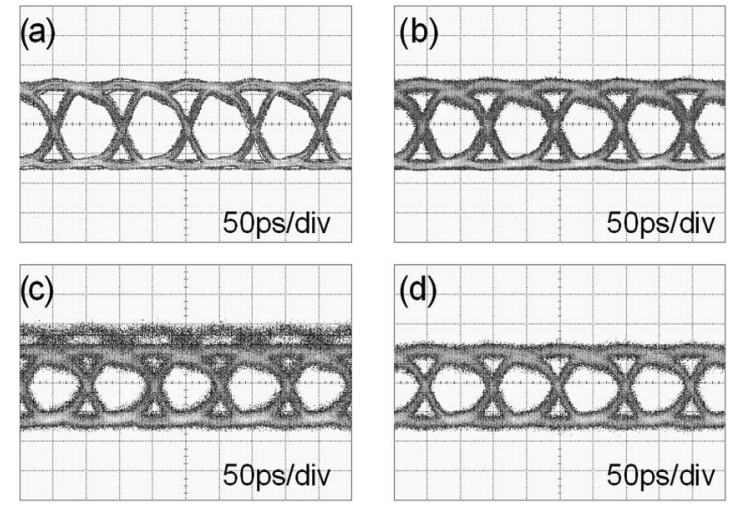

Fig. 8. Eye diagram of the payload signal at different points of the demonstrator. (a) Back-to-back signal, (b) after 40-km transmission, (c) after routing through the AWG, and (d) after routing without labeling.

and capturing of the label content. To prevent erroneous interpretation of the label content, commercial applications would require a cyclic redundancy check $(\mathrm{CRC})$ or more sophisticated error correction methods. For proof-of-concept of the STOLAS labeling technique, we have chosen to disregard this aspect to not unnecessarily complicate the design and keep focused on the label routing functionality. To facilitate the clock recovery, a preamble is added to the label. The clock recovery itself is based on slope detection using four clock phases each $90^{\circ}$ apart.

The combined FSK/IM optical signal was then boosted and launched into a dispersion compensated fiber link composed of $40 \mathrm{~km}$ of standard single mode fiber (SMF) followed by a matching length of $7 \mathrm{~km}$ of dispersion compensating fiber (DCF). Then, the signal was routed through the AWG and split: A portion was sent to an external optical add-drop multiplexer (OADM). In the OADM, FSK label detection is performed by optical filtering of one of the FSK tones followed by direct detection. The other signal portion was optically amplified and sent to a core router. The core router is composed of a fieldprogrammable gate array (FPGA) that performs the reading of the label from the OADM, generates the new label, and sets the integrated TLS (both its wavelength and the label word to be generated). An SOA-MZI module performs the wavelength conversion operation.

Fig. 8 shows the eye diagram of the signal at different points of the setup, namely, the payload signal in back-to-back configuration, after $40 \mathrm{~km}$ of SMF, after routing through the AWG, and after routing without labeling.

As it can be observed from Fig. 8, the signal suffers from intensity crosstalk from the label to the payload [Fig. 8(c)]. This transfer of optical power is due to a misalignment in the AWG central frequency with respect to the channel, resulting in an extra attenuation for one of the peaks of the label and, hence, fluctuations in the FSK signal. Fig. 8(d) shows the signal after the AWG when it has not been FSK labeled; in this case, the eye diagram remains wide open and clear.

Fig. 9 shows the bit-error-rate (BER) performance of the signal at the same points than in Fig. 8. The power penalty remains within a margin of $2 \mathrm{~dB}$, for a BER reference of $10^{-9}$, including the case when the signal is FSK labeled, and hence, there is crosstalk. In all cases, the ER remains around $6.5 \mathrm{~dB}$, and the FSK frequency deviation is fixed to $20 \mathrm{GHz}$.

Fig. 10 shows the eye diagram of the signal when it is wavelength converted to the other different channels. As it can be observed, the FSK-to-IM crosstalk generated at the AWG is converted to a new signal and can be seen at the eye diagram as a noise band. However, as Fig. 11 shows, this FSK-to-IM crosstalk affects the BER performance by only $1.5-\mathrm{dB}$ power penalty at a BER of $10^{-9}$. In conclusion, designing the system alignment of the channel frequency and the corresponding filter stages requires attention.

\section{A. Extinction Ratio (ER)}

Conventional IM systems aim for signals with high values of the ER, since this high relation between the ones and the zeros is generally translated into a better performance after transmission along optical fiber links. However, in a combined FSK/IM scheme, the value ER of the IM payload signal is compromised. Since the IM payload signal is superimposed on the FSK label signal, a high ER of the payload can lead to a bad performance of the label and vice versa. Fig. 12 shows how the receiver sensitivities for the IM payload and for the FSK label vary at the $50-\mathrm{Mb} / \mathrm{s}$ label rate when the payload ER is increased from 6 to $12 \mathrm{~dB}$. The label receiver sensitivity then degrades by $2 \mathrm{~dB}$, whereas the payload receiver sensitivity improves by more than $3 \mathrm{~dB}$. The optimum ER is found to be around $14 \mathrm{~dB}$. At the higher label rate of $312 \mathrm{Mb} / \mathrm{s}$, the degradation of the label receiver sensitivity with increasing ER is more pronounced, because less payload bits per label bit are available. As shown in Fig. 12, this yields a lower ER (about $6.5 \mathrm{~dB}$ ) and, hence, yields a degraded IM payload receiver sensitivity. Next to lowering the label rate, applying forward error correction techniques on the label data allows an increase of the ER and, thus, to improve the overall system performance [19]. Furthermore, it has also been experimentally demonstrated that by applying certain dc-balanced encoding such as Manchester [13], [14] or $8 \mathrm{~B} / 10 \mathrm{~B}$ [20], the crosstalk between the label and the payload can be reduced, hence allowing for the implementation of systems employing high values of the IM payload ER.

\section{B. Optical Filtering Issues}

Another important performance issue in a system consisting of a cascade of a number of optical nodes is the spectral misalignment between the central wavelength of the signal and the wavelength selective element of the nodes [22], [23]. This detrimental effect becomes stronger in the case of the combined FSK/IM scheme, where the broadening of the spectrum due to FSK modulation will not only disturb the signalfilter alignment, but it can also shift a portion of the signal spectrum outside the filter bandwidth, possibly leading to a serious 


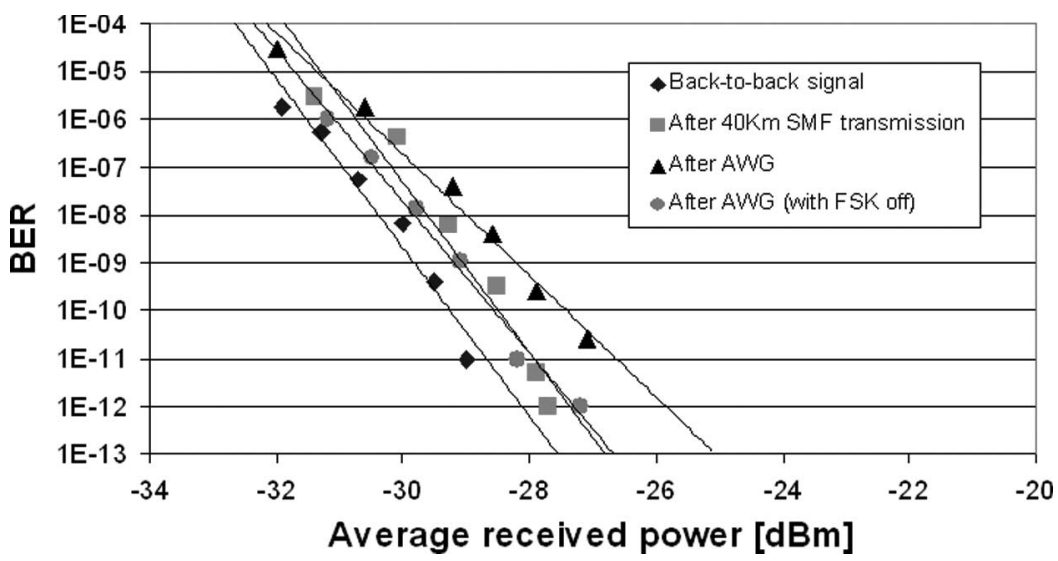

Fig. 9. BER performance of the payload signal back-to-back, after 40-km transmission over SMF, after being routed by an AWG, and after being routed by an AWG without an FSK label.

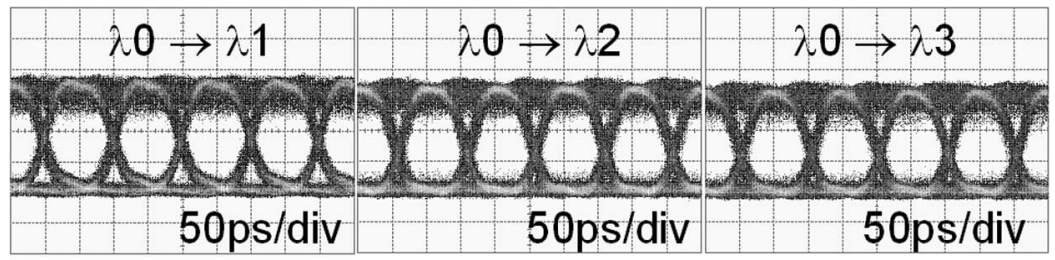

Fig. 10. Eye diagram of the signal after being wavelength translated to different channels.

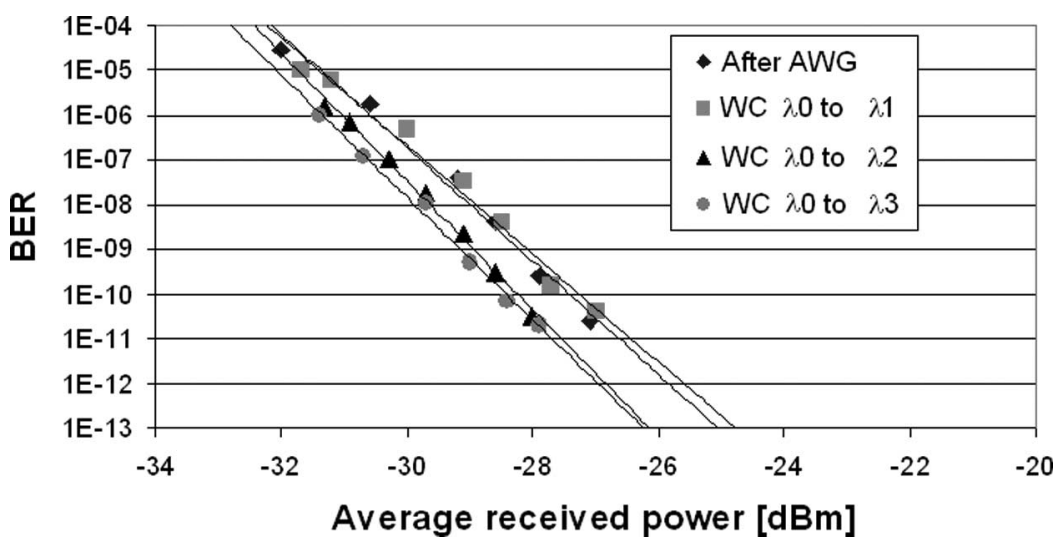

Fig. 11. BER performance of the payload after wavelength conversion to different channels.

signal degradation (crosstalk from the FSK to the IM signal) [24]-[26].

To investigate the effects of optical filtering, a simplified FSK/IM generator is simulated. A continuous wave $(\mathrm{CW})$ laser source $(192.50 \mathrm{THz})$ is FSK modulated with a frequency deviation of $20 \mathrm{GHz}$. This signal is subsequently modulated with an IM modulator with an ER of $6.5 \mathrm{~dB}$. The payload bit pattern is a $2^{31}-1$ PRBS NRZ at $10 \mathrm{~Gb} / \mathrm{s}$. The receiver is modeled as a photodiode, followed by a third-order Bessel low-pass filter. In order to simulate a filter with different wavelength shift, we used a tunable Gaussian-shaped optical band-pass filter (BPF) with an FWHM of $0.6 \mathrm{~nm}$. During the simulations, the opticalfilter center frequency is varied from 192.48 to $192.50 \mathrm{THz}$. Only the performance of the IM payload signal was measured at the output of the system. The results of the BER, as a function of the received optical power of the IM payload signal, are shown in Fig. 13. It shows BER curves for filter shift from 0 to $20 \mathrm{GHz}$ in steps of $2.5 \mathrm{GHz}$. In order to maintain a BER below $10^{-9}$, a maximum shift of the optical-filter center frequency of $7.5 \mathrm{GHz}$ is tolerable with respect to the center emission frequency. In this case, the power penalty is around $5.5 \mathrm{~dB}$.

In order to verify the prediction of the computer simulations, experiments on a similar scheme were performed. In the experimental setup, the receiver was composed of an EDFA providing a fix gain of $25 \mathrm{~dB}$ followed by a Gaussian-shaped optical BPF with an FWHM of $1.3 \mathrm{~nm}$ to suppress the amplified spontaneous emission (ASE) noise produced by the amplifier.

Fig. 14 shows the BER of the received IM signal with respect to the received power. A power penalty of $1.1(5 \mathrm{GHz}), 1.8$ $(10 \mathrm{GHz}), 2.8(15 \mathrm{GHz})$, and $4.1 \mathrm{~dB}$ for $20 \mathrm{GHz}$ was observed. For comparison purposes, the penalty in the case of $20-\mathrm{GHz}$ misalignment for the pure IM payload signal is just $0.4 \mathrm{~dB}$.

The slight performance improvement observed for the case of $0-\mathrm{GHz}$ shift over the back-to-back configuration due to the 


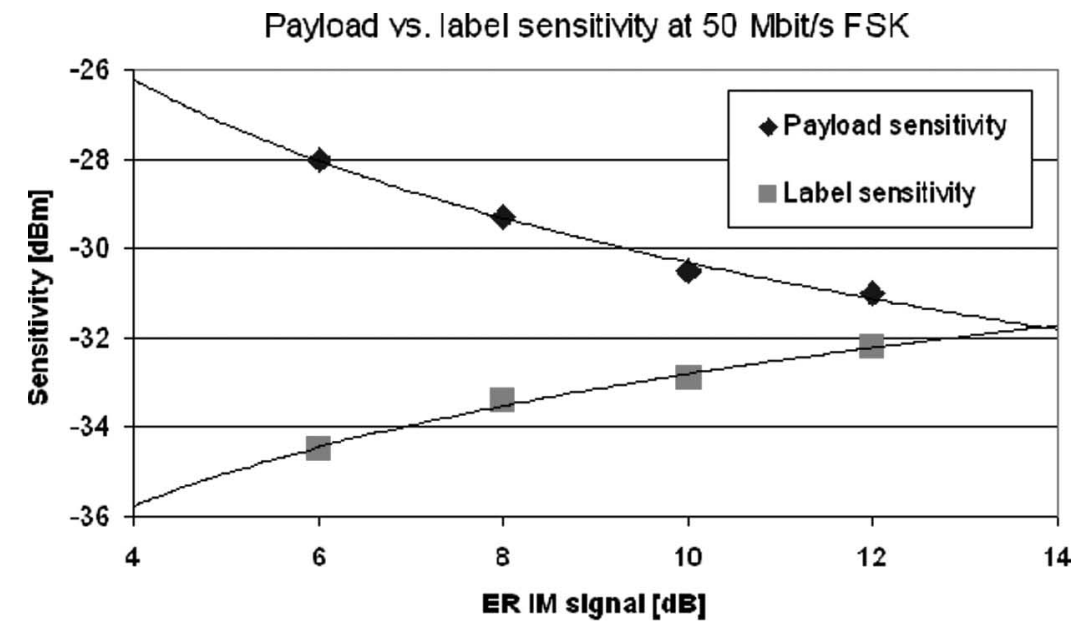

Payload vs. label sentivity at $320 \mathrm{mbit} / \mathrm{s}$ FSK

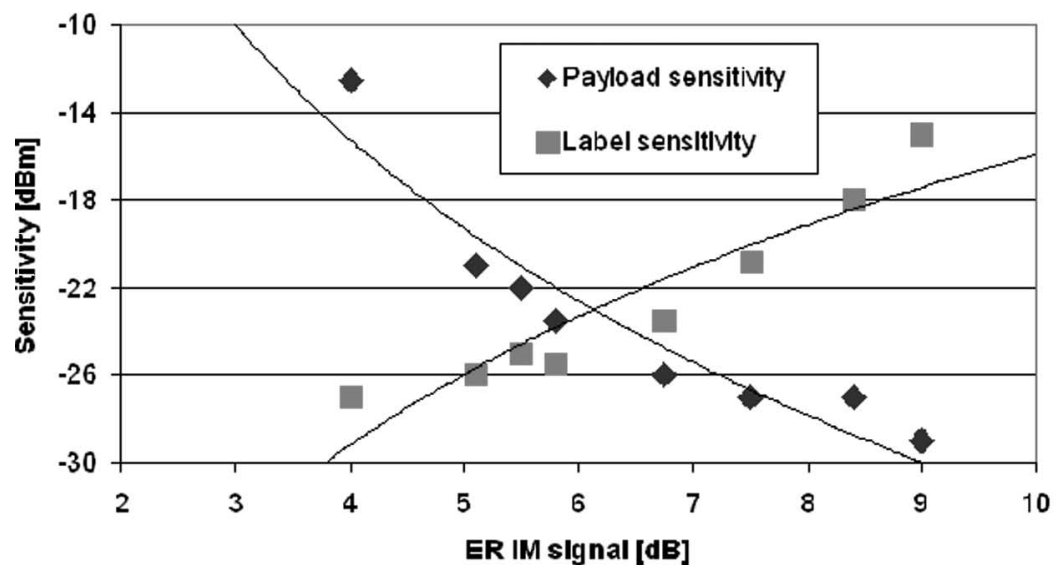

Fig. 12. Impact of payload ER at 50-Mb/s FSK label rate and 312-Mb/s FSK label rate.

introduction of the tunable filter reduces the ASE noise into the receiver.

The experimental results show that with a Gaussian-shaped optical, the permitted frequency misalignment between laser and filter is limited to $15 \mathrm{GHz}$, for a power penalty of the payload data below $3 \mathrm{~dB}$. The simulation results are more pessimistic and predicted a BER error floor for frequency shift above $7.5 \mathrm{GHz}$, while in the experimental results, a BER is observed for a frequency shift above $20 \mathrm{GHz}$.

\section{Wavelength Conversion}

In the considered optical network architecture, composed of a number of interconnected optical routing nodes, one of the main network impairments is the spectral misalignment between the central wavelength of the modulated signal and the wavelength selective element of the nodes, such as AWG-based optical filters. This spectral misalignment will inevitably increase the network susceptibility to power loss and interchannel optical crosstalk in the case of WDM transmission. From an intranode point of view, these misalignments may lead to interchannel and FSK-to-IM crosstalk effects in the wavelength converter, which is based on an SOA-MZI structure [21]. In this section, we experimentally studied the intranode crosstalk effect in an FSK/IM labeled system for different channel spacing of the interfering signals. In order to assess this effect, a setup

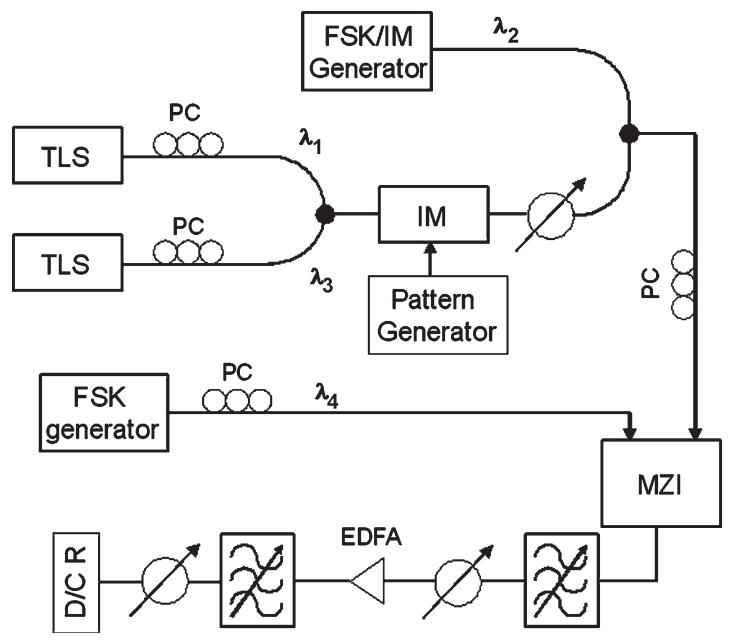

Fig. 13. BER curves for different filter shift.

simulating a central channel with two adjacent channels was implemented. The experimental setup is shown in Fig. 15.

An FSK/IM signal source, emitting at $1557.36 \mathrm{~nm}\left(\lambda_{2}\right)$, was modulated using a $10-\mathrm{Gb} / \mathrm{s} 2^{31}-1$ PRBS data stream. The hexadecimal label word "14" was used. The ER was set to be $6.68 \mathrm{~dB}$. Another two TLSs, emitting at 1555.75 and $1558.98 \mathrm{~nm}$, respectively, were modulated with a $10-\mathrm{Gb} / \mathrm{s}$ $2^{31}-1$ data stream and passed through a variable optical 


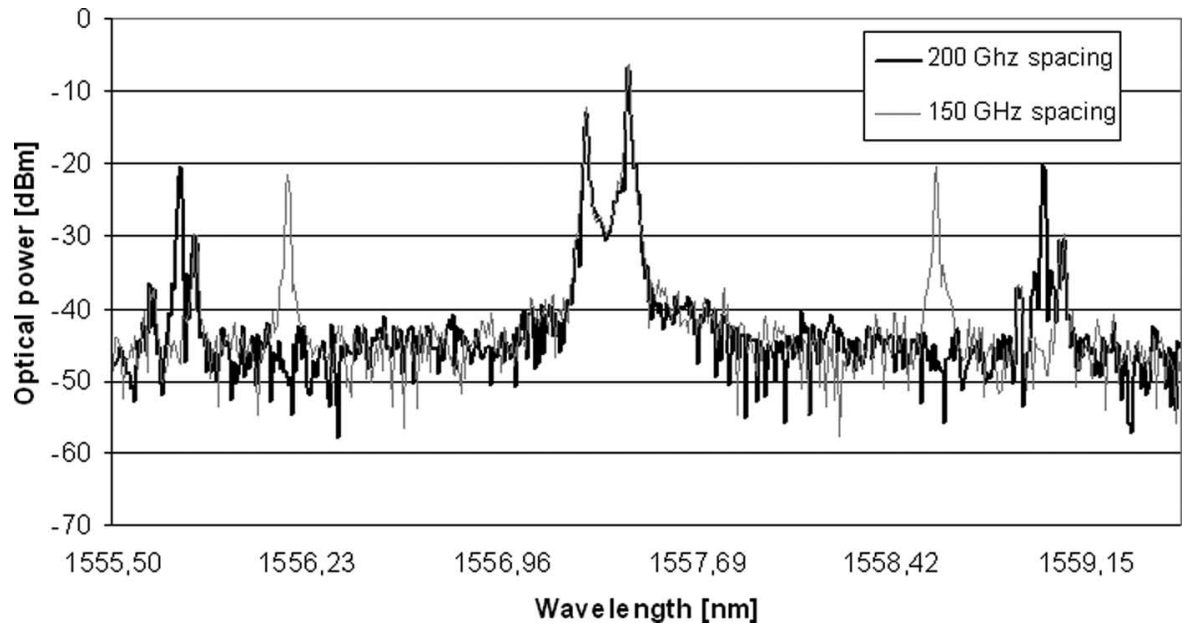

Fig. 14. Performance of filtered FSK/IM signal as a function of frequency detuning in a single-channel scenario.

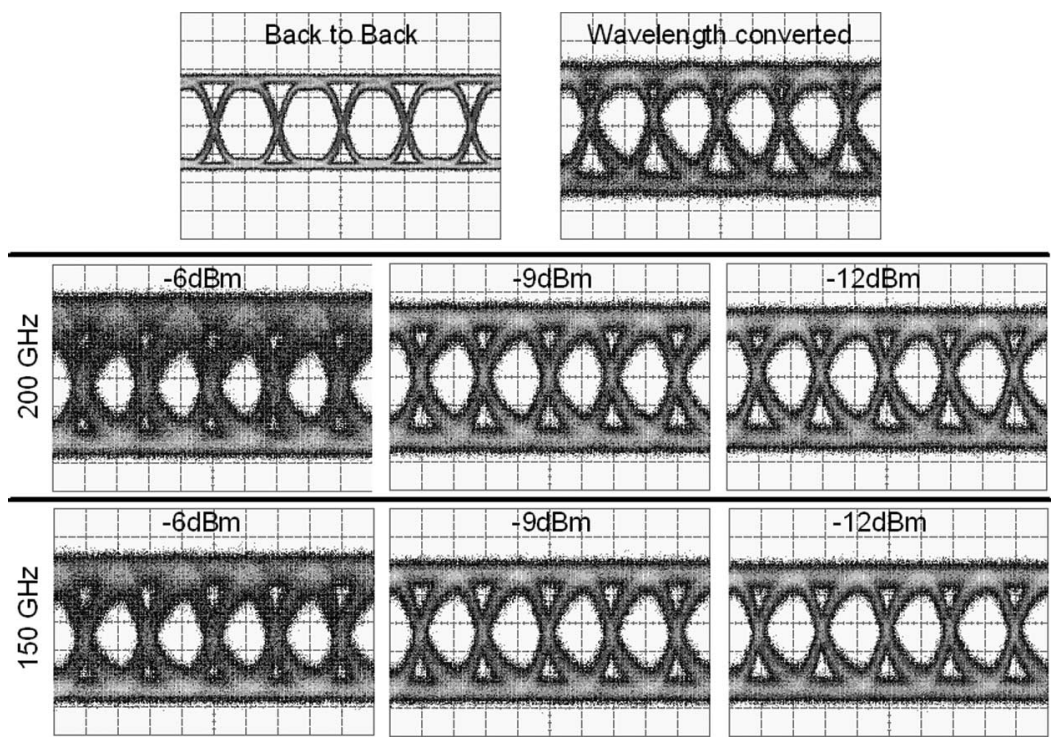

Fig. 15. Experimental setup. TLS: tunable laser source. IM: intensity modulator. PC: polarization controller. EDFA: erbium-doped fiber amplifier. D/C R: data/clock recovery.

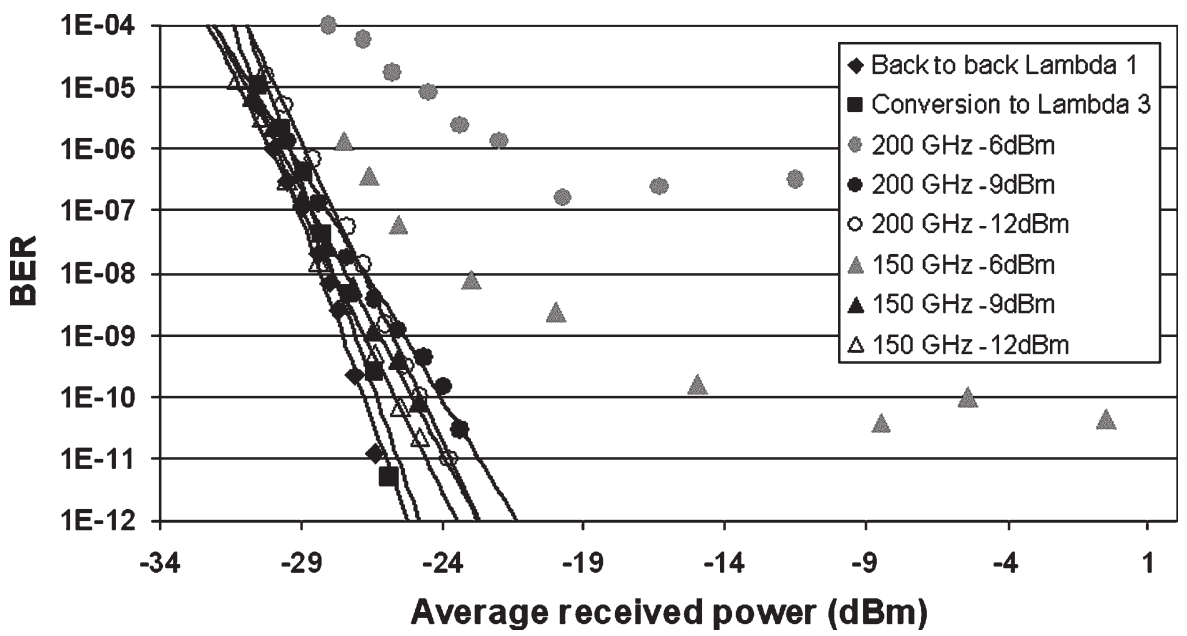

Fig. 16. Optical signal spectra of the FSK/IM labeled signal and interfering channels. 


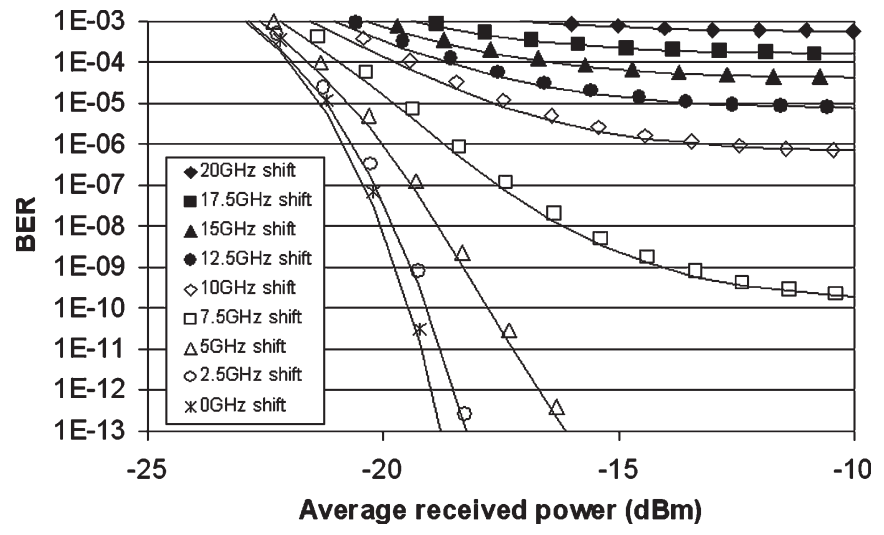

Fig. 17. Eye diagram of the back to back signal, the converted signal, and the converted signal with different levels of interfering signals for 150 - and 200-GHz spacing.

attenuator. Then, the signals were combined and sent to the SOA-MZI, which was operating in a copropagating scheme. The pumping signal to the MZI is a second FSK/IM generator, emitting at $1560.61 \mathrm{~nm}$. Therefore, in this system, the interfering signals were $200 \mathrm{GHz}$ away from the original signal, and the wavelength conversion performed over $400 \mathrm{GHz}$.

An optical BPF with 0.5-nm bandwidth at the output of the SOA-MZI filtered out the old signal and removed the ASE noise. Then, the signal was launched into the receiver block, consisting of an EDFA with a constant pumping current of $1000 \mathrm{~mA}$, an optical BPF $(0.5 \mathrm{~nm})$, and a data/clock recovery. The experiments were also performed when the TLS was tuned to 1556.16 and $1558.59 \mathrm{~nm}$, simulating a system where the interfering signals were $150 \mathrm{GHz}$ away from the original signal. Fig. 16 shows the optical signal spectra for both cases.

The obtained eye diagrams for the back-to-back case (without interfering signals) and with different levels of interfering signal power $(-6,-9$, and $-12 \mathrm{dBm})$ for two different channel spacings are shown in Fig. 17.

Fig. 18 shows the measured BER curves of the system. For the back-to-back case, we obtained receiver sensitivity at a BER of $10^{-9}$ of $-27.5 \mathrm{dBm}$. The power penalty in the interferencefree case was $0.5 \mathrm{~dB}$. The performance of the system in the presence of interfering signals is maintained below $2 \mathrm{~dB}$ of power penalty for interfering signal powers below $-9 \mathrm{dBm}$.

When the average power level of the interfering signals is above $-9 \mathrm{dBm}$ (in this case $-12 \mathrm{dBm}$ ), the power penalty increases substantially, and the converted signal is unrecoverable. The results demonstrate that by choosing the right operation point in the SOA-MZI, the tolerated average optical power level of interfering crosstalk might be, in general, up to $-10 \mathrm{dBm}$.

\section{Chirp Due to Wavelength Conversion in an SOA-MZI}

In this section, we focus on the frequency chirp properties of an SOA-MZI wavelength converter [27]. Since the chirp represents sudden variations in the frequency of the signal, any added chirp is of crucial importance for the performance of the FSK modulation format.

The employed chirp measurement method is based on a Fabry-Pérot interferometer, as a frequency discriminator [28].
The chirp measurements on the SOA-MZI wavelength converter were carried out for $2.5 \mathrm{~Gb} / \mathrm{s}$ as well as for $10 \mathrm{~Gb} / \mathrm{s}$. The results of the chirp measurement are listed in Table II.

For a noninverting conversion, we found a red shift (negative frequency shift) on the rising edge and a blue shift (positive frequency shift) on the falling edge. For inverting operation, the chirp is opposite in sign. This corresponds to the known theory that the chirp sign depends on the operation mode of the wavelength converter [29]. The peak-to-peak chirp measured is varying between 3.5 and $5 \mathrm{GHz}$. For both considered bit rates, no substantial differences in peak-to-peak chirp were observed. The ER of the converted signal can be tuned by polarization control of the pump signal and by adjusting the current settings. The chirp peaks of the converted signal are not dependent on the ER of the output signal or on the modulation speed. Two modulation techniques with different chirp properties are used for the modulation of the wavelength converter input signal, namely, direct modulation of a distributed feedback (DFB) laser and the usage of an external LiNbO3 modulator. The results show no substantial difference in the chirp of the output signal. This corresponds to the known fact that the chirp of the wavelength converted signal is primarily determined by the intensity of the modulated input signal and the device properties and not by the chirp of the input signal [30]. The input $\mathrm{CW}$ power (probe) for $10 \mathrm{~Gb} / \mathrm{s}$ is $1.7 \mathrm{dBm}$, and the input signal power (pump) is $P_{\text {in }}=-11.0 \mathrm{dBm}$ for noninverting operation and $P_{\text {in }}=-10 \mathrm{dBm}$ for inverting operation. For $2.5 \mathrm{~Gb} / \mathrm{s}$ inverting operation, the probe power is $+0.7 \mathrm{dBm}$ and $P_{\text {in }}=-10 \mathrm{dBm}$. An example of the time-resolved measurement of the chirp and the corresponding pulse is shown in Fig. 19.

The simulated chirp peak-to-peak values of the MZI wavelength converter are between 2 and $4 \mathrm{GHz}$, depending on the ER and power of the input signal. Since the chirp values are below $4 \mathrm{GHz}$, considering FSK modulation with frequency deviation in the range of $10-20 \mathrm{GHz}$, no substantial degradation of the FSK receiver performance is expected from SOA-MZI as wavelength converters for FSK/IM labeled signals.

\section{CASCADING OF FSK/IM LABEL SWAPPERS}

In order to assess the scalability of the label-swapping concept, experiments have been done with putting two labelswapping TWCs in cascade, using an ER of 7 and $12 \mathrm{~dB}$. Four wavelength channels were used, with a spacing of $200 \mathrm{GHz}(1555.75,1557.36,1558.98$, and $1560.61 \mathrm{~nm})$. The payload BER measurement results are shown in Fig. 20. Passing through a single TWC, a power penalty at BER $=10-9$ of $2.7 \mathrm{~dB}$ is incurred for an $\mathrm{ER}=7 \mathrm{~dB}$, and of $1.9 \mathrm{~dB}$ for $\mathrm{ER}=12 \mathrm{~dB}$. Passing two TWCs, the penalties are 5.3 and $4.4 \mathrm{~dB}$, respectively. These cumulative penalties are largely due to insufficient speed of the SOAs inside the TWC, which cause patterning effects. With a payload rate of $10 \mathrm{~Gb} / \mathrm{s}$ and a dynamic range of $20 \mathrm{~dB}$ for the payload receiver, the insufficient TWC speed limits the cascadability to four nodes. At a lower payload speed of $2.5 \mathrm{~Gb} / \mathrm{s}$, the penalties are found to be remarkably lower $(<2 \mathrm{~dB}$ after passing six nodes); hence, many more nodes could be cascaded. 


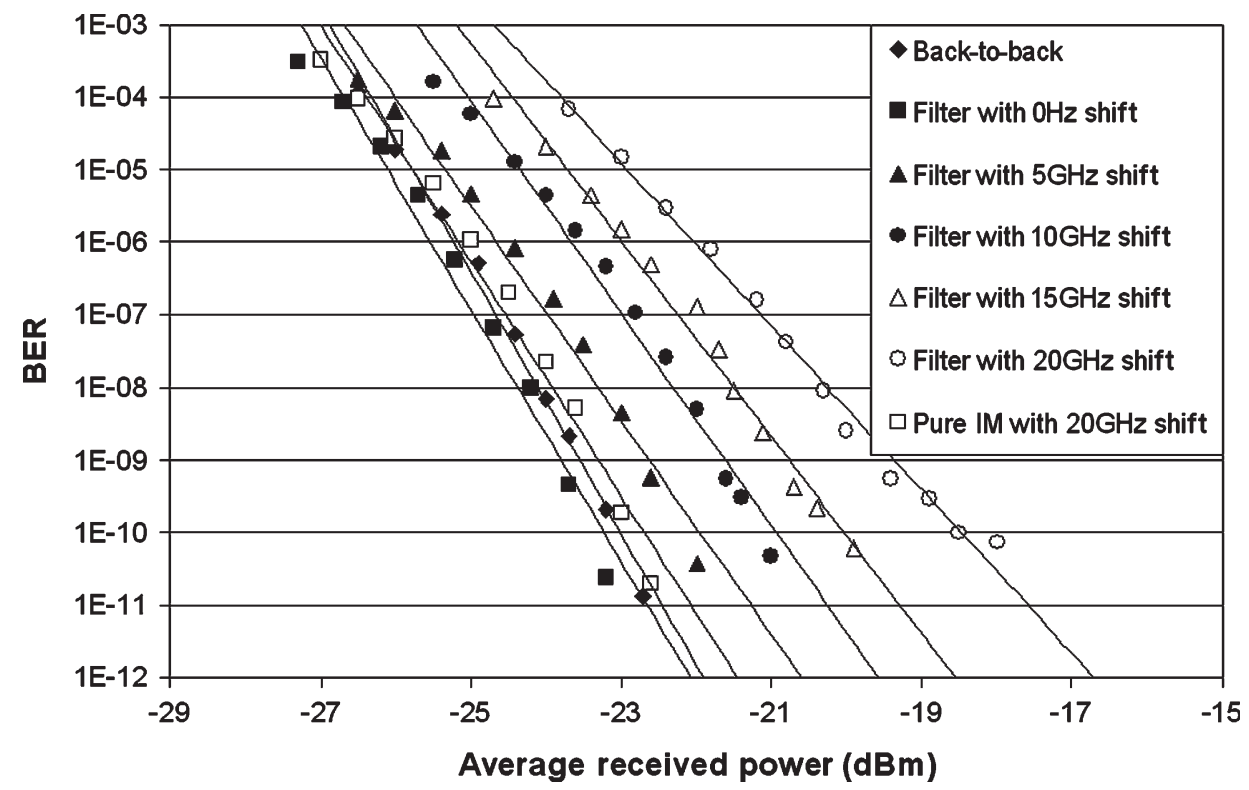

Fig. 18. BER curves of the payload signal for different levels of interfering signal at different channel spacing.

TABLE II

Chirp PeAKS FOr COPROPAGATING CONVERSION AT 2.5 AND $10 \mathrm{~Gb} / \mathrm{s} \mathrm{FOR}$ AN SOA-MZI WAVELENGTH CONVERTER

\begin{tabular}{|c|c|c|c|c|}
\hline $\begin{array}{l}\text { Bitrate } \\
(\mathrm{Gb} / \mathrm{s})\end{array}$ & $\begin{array}{c}\text { Chirp } \\
\text { (GHz) } \\
\text { Falling } \\
\text { edge }\end{array}$ & $\begin{array}{c}\text { Chirp } \\
\text { (GHz) } \\
\text { Rising } \\
\text { edge }\end{array}$ & Inv/Non-inv & $\operatorname{Pin}(\mathrm{dBm}$ \\
\hline 2.5 & -3.0 & 1.8 & Inv & -10.0 \\
\hline 10 & -2.1 & 1.5 & Inv & -10.0 \\
\hline 2.5 & 1.7 & -2.8 & Non-inv & -11.5 \\
\hline 10 & 1.7 & -2.2 & Non-inv & -11.0 \\
\hline
\end{tabular}

\section{ENGINEERING RULES}

In this section, we present a set of simple engineering rules for the design of a label-controlled routing node supporting the FSK/IM labeling scheme. The engineering rules are related to the main operations required in a label switched network, namely, to the labeling process, label detection, label swapping (including label erasure), wavelength conversion and rewriting of a new label, filtering issues, and node cascadability.

These rules are given for a system at $10-\mathrm{Gb} / \mathrm{s}$ payload, with an FSK label at low bit rate (namely, below $155 \mathrm{Mb} / \mathrm{s}$ ), in a WDM system with channels in agreement with the International Telecommunications Union (ITU) spacing of 100 and/or $200 \mathrm{GHz}$.

\section{A. FSK/IM Combined Signal}

Regarding the FSK/IM generation, we have the following statements.

1) The minimum payload length is determined by the length of the label signal.

2) The time synchronization of the label and payload is limited to ensure that the label signal is superimposed on top of the payload signal and not earlier than the start of

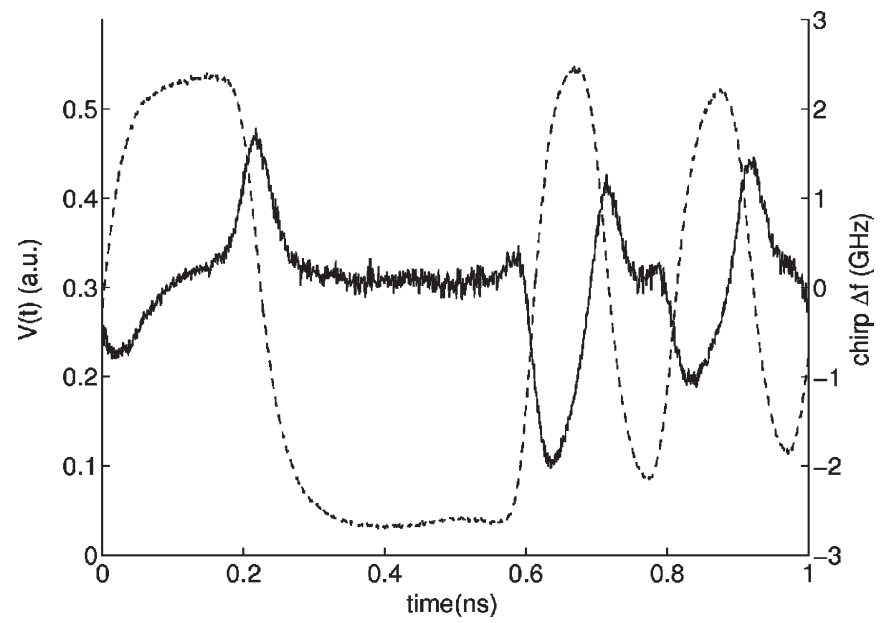

Fig. 19. Chirp measurement for $10 \mathrm{~Gb} / \mathrm{s}$ and noninverting operation. Dashed line is the pulse shape corresponding to the left $y$-axis, and the solid line is the chirp (gigahertz) corresponding to the right $y$-axis.

the payload and not later so that it exceeds the end of the payload section. The control block of the label swapper should hence be designed to detect both the FSK label and the start of the IM payload signal.

3) An important parameter in the combined FSK/IM signal is the choice of the ER for the IM signal, as seen in Section III-A. A value of the ER, providing the same receiver sensitivity for both signals, has been found to be in the range of 6-7 $\mathrm{dB}$ for a reference system operating at $155-\mathrm{Mb} / \mathrm{s}$ FSK and $10-\mathrm{Gb} / \mathrm{s}$ IM. The ER requirement could be relaxed if signal coding such as $8 \mathrm{~B} / 10 \mathrm{~B}$ or $64 \mathrm{~B} / 68 \mathrm{~B}$ is used or if a lower bit rate for the label data are employed. Forward equivalence class (FEC) codification for the label could also relax the IM ER. However, FEC codification should be applied in a higher layer of the system stack and not in the physical layer. 


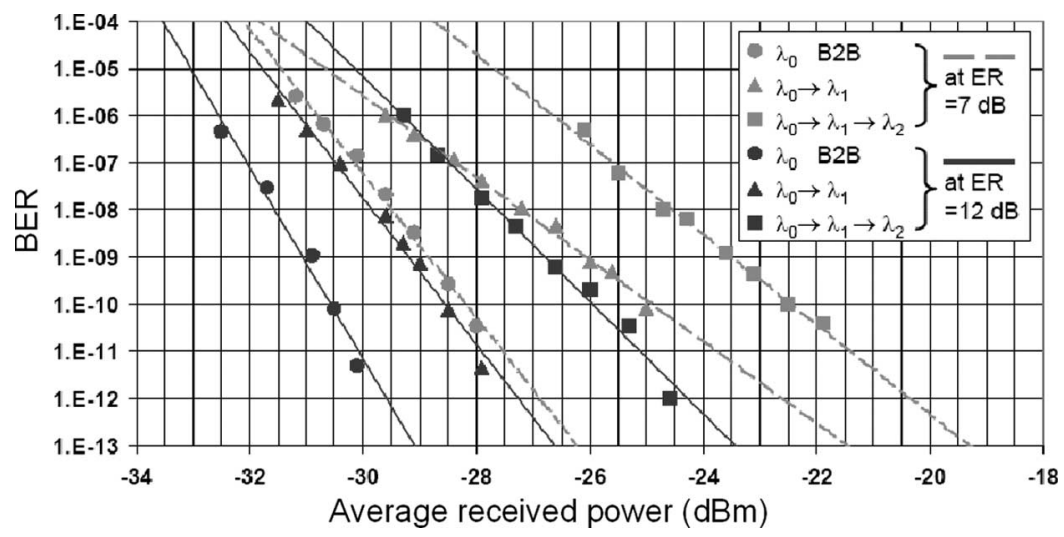

Fig. 20. Payload BER performance when cascading TWCs for ER $=7$ and $12 \mathrm{~dB}$.

4) A frequency deviation of $20 \mathrm{GHz}$ has been chosen for the laboratory trial. In this way, simple FSK detection is realized as well as allowing for tolerable wavelength drift of the TLSs. A smaller frequency deviation could be used; however, stricter optical filtering and wavelength stability will be required. If stability and precise optical filtering can be provided, lower frequency deviation allows higher FSK bit rates [31].

Label detection can be simply realized by a direct detection scheme using an optical BPF centered on one of the frequency tones of the FSK signal. A scheme using direct detection and a balanced receiver, where both FSK tones are filtered, is another alternative, resulting in improved receiver sensitivity. The following design choice is relevant for FSK/IM signal detection.

5) For an FSK label signal, the linewidth requirement of the TLS is not a limitation for its agility. For example, a laser linewidth of $100 \mathrm{MHz}$ does not show, according to simulations, any performance limitation on the FSK detection system at $155 \mathrm{Mb} / \mathrm{s} \mathrm{[32].}$

\section{B. FSK System and Filtering Issues}

Signals in an OLS network will encounter several stages of optical filtering. Therefore, special attention should be paid to the design regarding optical filtering, wavelength channel spacing, and FSK frequency deviation parameters, so that no residual FSK-to-IM modulation is introduced by filter shape or wavelength misalignment between the filter central wavelength and laser source emission wavelength. The considerations below, regarding optical filtering of the FSK/IM signal, relates to a $10-\mathrm{Gb} / \mathrm{s}$ IM payload data rate. It should be noticed that residual FSK-to-IM conversion is accumulated in a cascade of nodes and is not removed by the wavelength conversion stage during FSK/IM label swapping.

6) Computer simulations and experiments show that systems using a Gaussian-shaped optical BPF or AWG router (second-order Gaussian-shaped with a 3-dB bandwidth of $75 \mathrm{GHz}$ ) allow for a frequency misalignment between laser source and filter, limited to $15 \mathrm{GHz}$ for a power penalty of the payload data of less than $3 \mathrm{~dB}$. Flat-top- shaped optical filters are preferred over Fabry-Pérot or Gaussian-shaped filters [25], [26]. When using a more flat-top-shaped filter, the influence of filter misalignment is negligible for the same frequency misalignment of $15 \mathrm{GHz}$.

This indicates that with an FSK/IM modulation format, filters should have a flat passband in order to reduce FSKto-IM conversion. Considering an FSK frequency deviation of $15 \mathrm{GHz}$, it can be concluded that a 50-GHz ITU WDM system is feasible, provided the wavelength alignment discussed above is met.

Apart from the FSK-to-IM crosstalk, filter or signal misalignment can also lead to interchannel crosstalk, as discussed in Section III-B.

7) Experimental results demonstrate that for WDM systems with 150 and $200 \mathrm{GHz}$ of channel spacing, the FSK/IM can stand up to $-10 \mathrm{dBm}$ of interfering signals with a power penalty of less than $3 \mathrm{~dB}$ with respect to no presence of interchannel crosstalk.

\section{Label Swapping and Cascadability}

Wavelength converters based on an SOA-MZI configuration are attractive solutions for building FSK/IM label swapper modules due to their potential for integration on a single photonic chip. Moreover, label erasure and insertion can be performed in a single wavelength conversion stage, without compromising the payload data integrity [33].

However, the SOA-MZI should not introduce any patterning effects. Experimental results have shown that no signal degradation is observable due to chirp induced in the wavelength conversion stage.

8) The ER of the IM payload signal determines the sensitivity of the payload and the power penalty during the label insertion and wavelength conversion process in the SOA-MZI.

\section{CONCLUSION}

The throughput and efficiency of optical routing nodes can be enhanced by employing optical labeling of the payload information. The labeling is achieved by adding low-bit rate 
labels while keeping in the optical domain the high-bit rate payload information. By exploiting these moderate bit rate labels, optoelectronic modules can perform all the label processing work in the node. Combined angle and IM is a promising scheme for implementing the labeling of optical signals. For example, the FSK/IM labeling scheme presented in this paper offers advantageous features such as relaxed timing delineation between payload and label and ease of label erasure and rewriting of new labels. By using agile TLSs, with FSK modulation capability, wavelength converters, and passive wavelength routing elements, a scalable modular label-controlled router featuring high reliability can be built.

Several aspects of physical parameters of an FSK/IM combined modulation format labeling scheme within a routing node have been studied and presented in this paper. Optical filtering requires special care, since the combined FSK/IM scheme has a broader spectra than pure IM signals. However, an acceptable deviation of $15 \mathrm{GHz}$ from the central frequency can be tolerated for a power penalty of $3 \mathrm{~dB}$. The requirements on the limited ER for the IM signal can be relaxed at low bit rates of the label signal or alternatively by introducing data encoding. As shown from the laboratory trial results, the cascadability of the FSK/IM label-controlled nodes is mainly limited by the insufficient speed and patterning effects of the wavelength converters that can be overcome by the ongoing progress and development of high-speed SOA-MZI wavelength converters up to $40 \mathrm{~Gb} / \mathrm{s}$ and above. Optical labeling by using FSK/IM represents a simple and attractive way to implement hybrid optical circuit and burst switching in optical networks.

\section{ACKNOWLEDGMENT}

The authors would like to thank T. Mullane from Intune Technologies, C. Peucheret from COM Research Center, and R. Smets from Bell Labs Research for their contributions.

\section{REFERENCES}

[1] C. Qiao, "Labeled optical burst switching for IP-over-WDM integration," IEEE Commun. Mag., vol. 38, no. 9, pp. 104-114, Sep. 2000.

[2] D. J. Blumenthal et al., "All-optical label swapping networks and technologies," J. Lightw. Technol., vol. 18, no. 12, pp. 2058-2075, Dec. 2000.

[3] S. J. B. Yoo et al., "High-performance optical-label switching packet routers and smart edge routers for the next-generation Internet," IEEE J. Sel. Areas Commun., vol. 21, no. 7, pp. 1041-1051, Sep. 2003.

[4] I. Tafur Monroy, J. Zhang, N. Chi, P. V. Holm-Nielsen, C. Peucheret, A. M. J. Koonen, J. J. Vegas Olmos, and G.-D. Khoe, "Techniques for labeling of optical signals in burst switched networks," in Proc. 1st Workshop on Optical Burst Switching, Opticomm, Dallas, TX, 2003, pp. 1-11.

[5] G. K. Chang and J. Yu, "Multi-rate payload switching using a swappable optical carrier suppressed label in a packet switched DWDM optical network," J. Lightw. Technol., vol. 23, no. 1, pp. 196-202, Jan. 2005.

[6] G. K. Chang, J. Yu, A. Chowdhury, and Y. K. Yeo, "Optical carrier suppression and separation label-switching techniques," J. Lightw. Technol., vol. 23, no. 10, pp. 3372-3387, Oct. 2005.

[7] C. W. Chow, C. S. Wong, and H. K. Tsang, "All-optical ASK/DPSK labelswapping and buffering using Fabry-Pérot laser diodes," IEEE J. Sel. Topics Quantum Electron., vol. 10, no. 2, pp. 363-370, Mar./Apr. 2004.

[8] W. Hung, C. K. Chan, L. K. Chen, and F. Tong, "A bit-serial optical packet label-swapping scheme using DPSK encoded labels," IEEE Photon. Technol. Lett., vol. 15, no. 11, pp. 1630-1632, Nov. 2003.

[9] C. W. Chow, C. S. Wong, and H. K. Tsang, "All-optical data-format and wavelength conversion in two-wavelength injection locked slave Fabry-Pérot laser diodes," Electron. Lett., vol. 39, no. 13, pp. 997-999, Jun. 2003.
[10] T. Koonen, G. Morthier, J. Jennen, H. deWaardt, and P. Demeester, "Optical packet routing in IP-over-WDM networks deploying two-level optical labeling," in Proc. ECOC, 2001, vol. 4, pp. 608-609.

[11] K. G. Vlachos, I. T. Monroy, A. M. J. Koonen, C. Peucheret, and P. Jeppesen, "STOLAS: Switching technologies for optically labeled signals," IEEE Commun. Mag., vol. 41, no. 11, pp. 43-49, Nov. 2003.

[12] A. M. J. Koonen, J. J. Vegas Olmos, I. Tafur Monroy, J. G. J. Jennen, C. Peucheret, and E. van Breusegem, "Optical packet routing using orthogonal labeling-Results from the FP5 STOLAS project," presented at the European Conf. Optical Communication, Glasgow, U.K., 2005, Mo.4.4.1.

[13] J. Zhang, N. Chi, P. V. Holm-Nielsen, C. Peucheret, and P. Jeppesen, "10 Gb/s Manchester-encoded FSK-labelled optical signal transmission link," Electron. Lett., vol. 39, no. 16, pp. 1193-1194, Aug. 7, 2003.

[14] - "Performance of Manchester-coded payload in an optical FSK labeling scheme," IEEE Photon. Technol. Lett., vol. 15, no. 8, pp. 1174-1176, Aug. 2003.

[15] S. Gao et al., "Wavelength requirements and routing for multicasting connections in lightpath and light-tree models of WDM networks with limited drops," Proc. Inst. Electr. Eng., Commun., vol. 148, no. 6, pp. 363367, 2001.

[16] O. A. Lavrova and D. J. Blumenthal, "Detailed transfer matrix based dynamic model for multisection widely-tunable GCSR lasers," J. Lightw. Technol., vol. 18, no. 9, pp. 1274-1283, Sep. 2000.

[17] K. Vlachos, J. G. L. Jennen, Y. Yu, and R. O'Dowd, “Optical FSK transmitter using widely tunable GCSR lasers," in Proc. CLEO/Eur.-EQEC, Munich, Germany, Jun. 23-27, 2003, p. 186.

[18] Y. Yu, G. Mulvihill, S. O'Duill, and R. O'Dowd, "Performance implications of wide-band lasers for FSK modulation labeling scheme," IEEE Photon. Technol. Lett., vol. 16, no. 1, pp. 39-41, Jan. 2004.

[19] I. Tafur Monroy, M. Martinez Chisvert, J. J. Vegas Olmos, and A. M. J. Koonen, "Error-correcting coding for FSK/IM modulation format for optical labeling of signals," in Proc. ONDM, Gent, Belgium, Feb. 2004, pp. 381-388.

[20] J. Zhang, P. V. Holm-Nielsen, N. Chi, C. Peucheret, and P. Jeppesen, "DC-balanced line encoding for optical labeling scheme using orthogonal modulation," presented at the Optical Fiber Communications Conf., Anaheim, CA, 2004, WF2.

[21] J. J. Vegas Olmos, I. Tafur Monroy, and A. M. J. Koonen, "Experimental assessment of intra-node crosstalk in a labelled system based on MZI wavelength converters," in Proc. NOC, London, U.K., Jul. 2005, pp. 407-414.

[22] N. Khrais, A. Elrefaie, R. Wagner, and S. Ahmed, "Performance degradation of multiwavelength optical networks due to laser and (de)multiplexer misalignments," IEEE Photon. Technol. Lett., vol. 7, no. 11, pp. 13481350, Nov. 1995.

[23] C. Caspar, H. Foisel, R. Freund, U. Kruger, and B. Strebel, "Cascadability of arrayed-waveguide grating (de)multiplexers in transparent optical networks," presented at the Optical Fiber Communications Conf., pp. 19-20, Dallas, TX, 1997, Paper TUE2.

[24] J. J. Vegas Olmos, I. Tafur Monroy, E. Tangdiongga, J. P. A. van Berkel, A. M. J. Koonen, and J. Prat, "Influence of optical filters on the performance of FSK/IM transmission schemes," in Proc. Opnetec, Pisa, Italy, Oct. 18-20, 2004, pp. 364-370.

[25] J. P. A. van Berkel, J. J. Vegas Olmos, I. Tafur Monroy, and A. M. J. Koonen, "Effect of non-optimal filtering on the performance of FSK/IM transmission schemes," in Proc. NOC, London, U.K., Jul. 5-7, 2005, pp. $423-430$.

[26] J. van Berkel, J. J. Vegas Olmos, I. Tafur Monroy, and A. M. J. Koonen, "Influence of non-optimal filtering on FSK/IM modulated signals," presented at the European Conf. Optical Communication, Glasgow, U.K., 2005, Th3.4.5.

[27] E. J. M. Verdurmen, A. M. J. Koonen, H. de Waardt, and I. Tafur Monroy, "Chirp properties of SOA-based wavelength converters for FSK/IM combined modulation format," presented at the European Conf. Optical Communication, Rimini, Italy, 2003, Paper TU4.4.5.

[28] S. Tammela, H. Ludvigsen, T. Kajava, and M. Kaivola, "Time-resolved frequency chirp measurement using a silicon-wafer etalon," IEEE Photon. Technol. Lett., vol. 9, no. 4, pp. 475-477, Apr. 1997.

[29] T. Durhuus, B. Mikkelsen, C. Joergensen, S. L. Danielsen, and K. E. Stubkjaer, "All-optical wavelength conversion by semiconductor optical amplifiers," J. Lightw. Technol., vol. 14, no. 6, pp. 942-954, Jun. 1996.

[30] S.-C. Cao and J. C. Cartledge, "Characterization of the chirp and intensity modulation properties of an SOA-MZI wavelength converter," J. Lightw. Technol., vol. 20, no. 4, pp. 689-695, Apr. 2002. 
[31] J. Prat, V. Polo, C. Bock, C. Arellano, and J. J. Vegas Olmos, "Full-duplex single fiber transmission using FSK downstream and IM remote upstream modulations for fiber-to-the-home," IEEE Photon. Technol. Lett., vol. 17, no. 3, pp. 702-704, Mar. 2005.

[32] K. I. Vlachos, J. Zhang, J. Cheyns, Sulur, N. Chi, E. Van Breusegem, I. Tafur Monroy, J. G. L. Jennen, P. V. Holm-Nielsen, C. Peucheret, R. O'Dowd, P. Demeester, and A. M. J. Koonen, "An optical FSK/IM coding technique for the implementation of a label-controlled arrayed waveguide packet router," J. Lightw. Technol., vol. 21, no. 11, pp. 26172628, Nov. 2003.
[33] J. J. Vegas Olmos, J. Zhang, P. V. Holm-Nielsen, I. Tafur Monroy, V. Polo, A. M. J. Koonen, C. Peucheret, and J. Prat, "Simultaneous optical label erasure and insertion in a single wavelength conversion stage of combined FSK/IM modulated signals," IEEE Photon. Technol. Lett., vol. 16, no. 9 , pp. 2144-2146, Sep. 2004.

Photographs and biographies of the authors were not available at the time of publication. 